\title{
Probing the electronic properties and charge state of gold nanoparticles on ultrathin MgO versus thick doped $\mathrm{CaO}$ films
}

\author{
Yi Cui, ${ }^{1}$ Christian Stiehler, ${ }^{1}$ Niklas Nilius,,${ }^{1,2, *}$ and Hans-Joachim Freund ${ }^{1}$ \\ ${ }^{1}$ Fritz-Haber-Institut der Max-Planck-Gesellschaft, Faradayweg 4-6, 14195 Berlin, Germany \\ ${ }^{2}$ Carl von Ossietzky Universität Oldenburg, Institut für Physik, D-26111 Oldenburg, Germany \\ (Received 29 May 2015; revised manuscript received 16 July 2015; published 28 August 2015)
}

\begin{abstract}
Electron transfer into metal nanoparticles on oxide supports is associated with unusual morphological, electronic, and chemical properties of the charged system. Two fundamental charging routes have been identified so far, which are electron tunneling through ultrathin oxide films supported by a bulk metal and charge donation from single-ion impurities embedded in the oxide matrix. In this study, we have investigated whether both routes lead to the formation of metal deposits with identical properties. For this purpose, Au islands have been prepared on 1-2 ML thin $\mathrm{MgO} / \mathrm{Ag}(001)$ layers and on 25-ML-thick $\mathrm{CaO}$ films doped with Mo impurities. The morphological and electronic properties of the islands were analyzed with low-temperature scanning tunneling microscopy (STM) and spectroscopy. In both systems, pronounced electron confinement effects are observed in the nanostructures, arising from the quantization of one and the same Au electronic band. Moreover, clear experimental signatures for a charge transfer into the islands are found, such as a layer-by-layer growth of the ad-metal and a negative contrast of the oxide region around the deposits in STM images. Our data provide evidence that the charged nanostructures exhibit comparable properties independent of the origin of the extra electrons. This agreement suggests that ultrathin oxide films may be used as model systems for doped bulk oxides, as used in heterogeneous catalysis.
\end{abstract}

DOI: 10.1103/PhysRevB.92.075444

PACS number(s): 73.21.Fg, 73.61.Ng, 68.65.Fg, 68.37.Ef

\section{INTRODUCTION}

The charge state of oxide-supported metal nanoparticles largely governs their physical and chemical properties [1,2]. As demonstrated by experiment [3] and theory [4,5], the presence of excess electrons controls the growth morphology of the ad-metal. While neutral deposits follow a three-dimensional (3D) growth regime due to their weak adhesion to the oxide support [6], two-dimensional (2D) islands preferentially develop after electron transfer into the ad-metal [3]. This surprising effect is explained with a reinforced metal-oxide interaction, arising from direct Coulomb attraction and chargemediated polarization effects of the oxide surface $[7,8]$. The charge transfer also alters the electronic structure of the metal deposits. The extra electrons occupy states around the Fermi level, which are preferentially located at low-coordinated edge and corner atoms of the nanostructure [9]. This specific distribution maximizes the spatial separation between the excess charges, which in turn minimizes their mutual repulsion. As a result, nanostructures with linear (1D) and planar (2D) shapes, exposing an abundance of low-coordinated sites, were found to be thermodynamically preferred upon charge transfer $[10,11]$. Also, the many-body response, e.g., the nature of plasmonic excitations, depends on the number and spatial distribution of extra charges in confined electronic systems [12].

However, the largest impact of the excess electrons is on the chemical properties of oxide-supported nanoparticles. Both the availability of low-coordinated sites and the easiness of redox processes promote chemical processes on the charged deposits [13]. In fact, pancake-shaped islands often exhibit a higher reactivity than their $3 \mathrm{D}$ counterparts, reflecting the abundance

*Corresponding author: niklas.nilius@uni-oldenburg.de of interface sites between metal and oxide surface [14,15]. Prominent examples are the efficient $\mathrm{CO} \rightarrow \mathrm{CO}_{2}$ conversion on bilayer $\mathrm{Au}$ deposits on $\mathrm{FeO}$ and $\mathrm{TiO}_{2}$ thin films [14,16], and the $\mathrm{CO}_{2}$ activation to oxalate on monolayer $\mathrm{Au}$ islands on $\mathrm{MgO}$ [17]. In general, the preparation of metal particles in a specific charge state represents one fundamental goal in catalysis-related research on oxide surfaces.

In recent years, two reliable routes have been developed for the preparation of charged nanoparticles. In the first one, the bulk oxide is replaced by a thin film grown on a metal support, as this configuration enables direct charge transfer through the insulating layer $[3,11]$. The efficiency and direction of the electron flow is hereby controlled by the Fermi energy in the substrate with respect to the affinity level of the adsorbates, and by the thickness and conductivity of the oxide spacer [18]. As most oxides reduce the work function of the underlying metal [19], electrons tend to flow towards the surface and trigger a negative charging of molecules and metal deposits. While electron exchange through an oxide spacer is limited to ultrathin films, the second route is accessible for oxides of arbitrary thickness. In this case, extra charges are provided by aliovalent dopants that have been introduced into the oxide matrix. The insertion of donor-type ions gives rise to the formation of negatively charged adsorbates $[5,20]$, while acceptors in the lattice promote a charge flow out of the ad-species [21]. The desired charging direction as well as the number of transfer electrons can thus be conveniently adjusted via the doping procedure.

In this paper, we aim to compare the two approaches of charge manipulation and therefore go beyond earlier work of our group that focused on electron transfer in one or the other limit $[9,10,20,22]$. The two model systems studied here are (i) bilayer $\mathrm{MgO} / \mathrm{Ag}(001)$ films that are transparent for electrons, and (ii) relatively thick $\mathrm{CaO}$ films doped with $\mathrm{Mo}$ ions. The dopants in the latter case enter the oxide from the 
Mo(001) substrate via temperature-controlled diffusion [23]. The relevance of charge-transfer processes is derived from the shape and electronic structure of Au nanoparticles on the oxide surfaces, as studied with scanning tunneling microscopy (STM) and spectroscopy [7]. While the properties of the $\mathrm{Au}$ islands seem to differ at first glance, we demonstrate that charge transfer takes place in both cases and leads to a comparable response of the ad-particles. Apparently, the source of electrons, either from the metal below the film or from donor-type impurities inside the oxide matrix, is irrelevant for the occurrence of charging effects in the nanogold.

\section{EXPERIMENTS}

The experiments have been performed in two, custom-built STM setups, operated in ultrahigh vacuum and at liquid helium temperature $(5-10 \mathrm{~K})$. Bilayer $\mathrm{MgO}$ films were prepared by reactive $\mathrm{Mg}$ deposition onto a sputtered and annealed $\mathrm{Ag}(001)$ surface at $1 \times 10^{-6} \mathrm{mbar}_{2}$ and $550 \mathrm{~K}$ sample temperature [24]. No postannealing was applied given the low thermal stability of the $\mathrm{Ag}$ support. $\mathrm{CaO}$ films of $25 \mathrm{ML}$ thickness were grown by room-temperature Ca deposition onto clean $\mathrm{Mo}(001)$ in $1 \times 10^{-6} \mathrm{mbar}_{2}$ [23]. Subsequent vacuum annealing to $1000 \mathrm{~K}$ triggered the formation of rocksalt $\mathrm{CaO}$ that, similar to $\mathrm{MgO}$, exposed the low-index (001) plane. During annealing, Mo atoms from the support spontaneously diffuse into the film, resulting in a deceasing Mo dopant concentration from $25 \%$ at the interface to nearly zero at the surface of a $25 \mathrm{ML}$ film [25]. Most of the Mo impurities occupy Ca substitutional sites and adopt a $2+$ or $3+$ charge state in the rocksalt lattice. As 6+ is the highest Mo oxidation state, the impurities are susceptible to electron transfer either into morphological trap sites of the lattice or into adsorbates on the oxide surface $[20,26]$.

Structure and morphology of the two oxides have been extensively studied with low-energy electron diffraction and STM before [23-25,27]. On their surfaces, the expected square arrangement of atoms with $3.0 \AA(\mathrm{MgO})$ and $3.4 \AA(\mathrm{CaO})$ periodicity is resolved, indicating the development of bulk-cut terminations. The films are characterized by atomically flat terraces separated by dislocation lines; the latter being inserted to release interfacial lattice strain. Terrace sizes were found to be $50 \%$ larger on $\mathrm{CaO}(001)$, reflecting the beneficial effect of postannealing. In a last preparation step, $0.05 \mathrm{ML}$ of gold was deposited onto the films at $300 \mathrm{~K}$. On both oxides, the Au atoms agglomerate into monolayer islands of $5-10 \mathrm{~nm}$ diameter. While on $\mathrm{CaO}$, most of the $\mathrm{Au}$ islands are of hexagonal shape (Fig. 1), a variety of configurations is found on $\mathrm{MgO}$ (Fig. 2) [28]. The difference reflects the lower surface quality and larger defect density of the low-temperature $\mathrm{MgO}$ layers.

\section{RESULTS AND DISCUSSION}

\section{A. Morphology of the Au nanostructures}

Our study focuses on the electronic properties and charge state of $\mathrm{Au}$ nanostructures grown on ultrathin $\mathrm{MgO}$ and thick, doped $\mathrm{CaO}$ films. To ensure comparability, we have selected Au islands of similar geometry. The target island has hexagonal shape, is roughly $5-7 \mathrm{~nm}$ in diameter, and contains 200-400 atoms (Figs. 1 and 2). As revealed from atomically resolved images, its lattice is of (111) type and therefore deviates from the square symmetry of the oxide surface [Fig. 2(c)]. The $\mathrm{CaO}$-supported islands exhibit a parallel stripe pattern with $7.5 \AA$ periodicity that appears in three orientations rotated by $120^{\circ}$ [25]. The pattern is ascribed to a hexagonal-on-square moiré structure, in which six [112]-oriented Au rows overgrow three $\mathrm{CaO}$ [100] unit cells [Fig. 1(c)]. The crests of the pattern are formed by $\mathrm{Au}$ atoms on $\mathrm{Ca}$ top sites, while the valleys are made of $\mathrm{Au}$ atoms in $\mathrm{O}$ top and hollow positions. As each moiré cell contains two crests, the distance between adjacent stripes $(7.5 \AA)$ is only half the unit cell length $(15 \AA)$. No superstructure is observed on $\mathrm{MgO}$-supported $\mathrm{Au}$ islands, for which the theoretical moiré periodicity would be $20 \AA$, corresponding to eight $\mathrm{Au}[112]$ rows overgrowing five $\mathrm{MgO}$ unit cells (Fig. 2). The stripe pattern might be invisible because its periodicity comes close to the diameter of the ad-islands. Moreover, density functional theory calculations found a rather flat potential landscape for $\mathrm{Au}$ adsorption on $\mathrm{MgO} / \mathrm{Ag}(001)$ films, characterized by similar binding parameters for the different $\mathrm{Mg}$ and $\mathrm{O}$ top and hollow positions [7].

Differences between the $\mathrm{CaO}$ and $\mathrm{MgO}$ support become evident not only in the internal structure of the Au islands, but also in their appearance with respect to the surrounding oxide. On both films, the nanogold undergoes a contrast reversal from a protruding entity at negative and small positive bias to

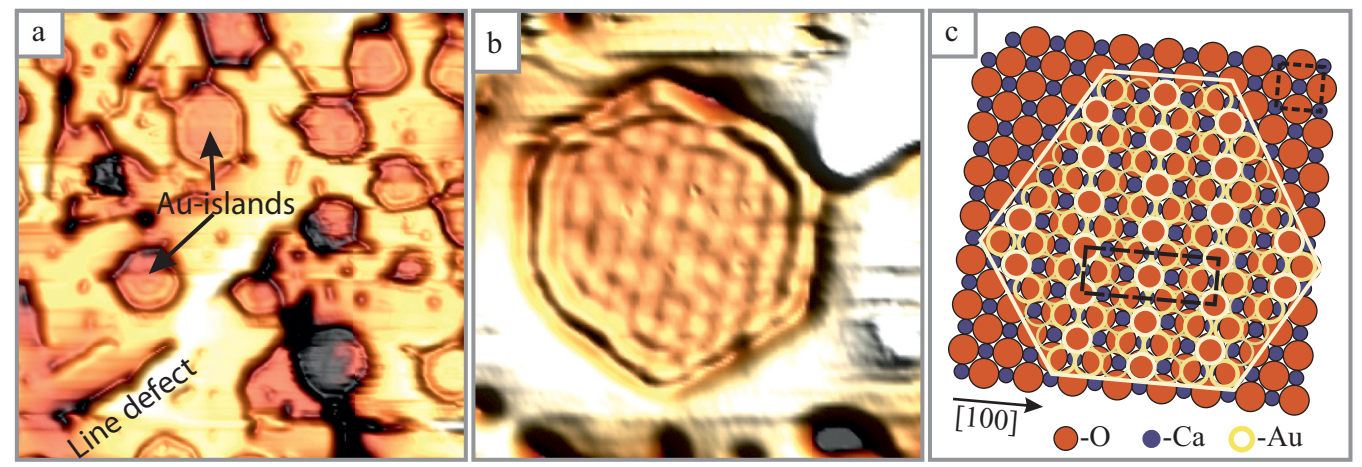

FIG. 1. (Color online) STM images in pseudo-3D representation of (a) several $\left(40 \times 40 \mathrm{~nm}^{2}\right)$ and (b) a single Au monolayer island $\left(11 \times 11 \mathrm{~nm}^{2}\right)$ grown on a 25-ML-thick, Mo-doped $\mathrm{CaO}$ film $\left(V_{s}=4.0 \mathrm{~V}, 15 \mathrm{pA}\right)$. The stripes in $(\mathrm{b})$ arise from a moiré pattern formed between the hexagonal $\mathrm{Au}(111)$ and the square $\mathrm{CaO}(001)$ lattices, as depicted in (c). 


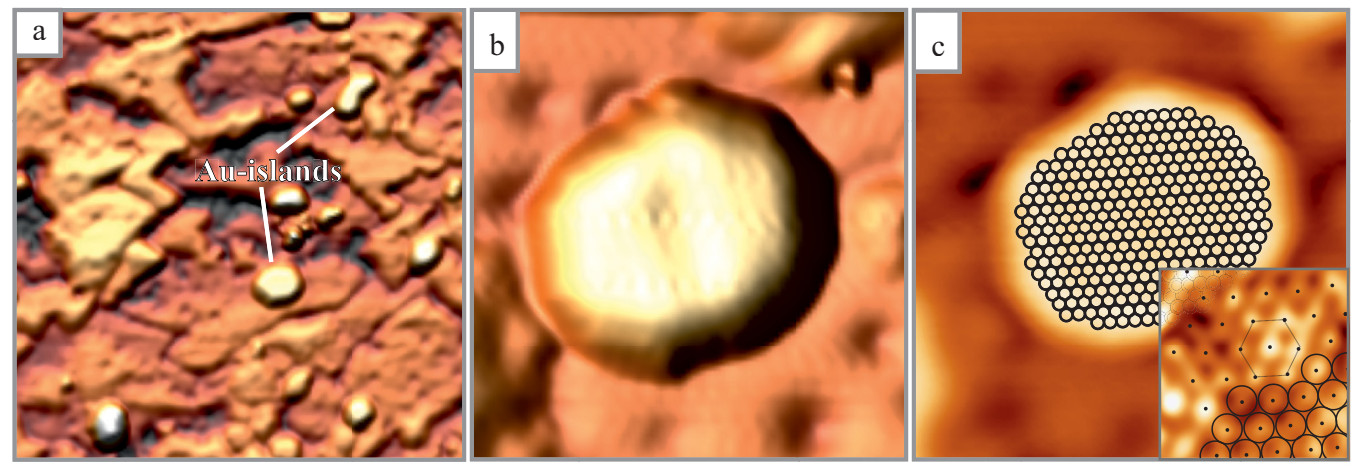

FIG. 2. (Color online) STM images of (a) several $\left(70 \times 70 \mathrm{~nm}^{2}\right)$ and (b) a single Au monolayer island $\left(11 \times 11 \mathrm{~nm}^{2}\right)$ on $2 \mathrm{ML} \mathrm{MgO} / \mathrm{Ag}(001)$ $\left(V_{s}=1.7 \mathrm{~V}, 15 \mathrm{pA}\right)$. (c) Filling of island (b) with a hexagonal lattice of $2.89 \AA$ A periodicity, as in bulk gold, gives an approximate atom number of 319. The inset displays the hexagonal symmetry of Au atoms in the top facet $\left(2 \times 2 \mathrm{~nm}^{2}\right)$.

a faint depression at elevated positive voltage (Fig. 3). Bright appearance of the islands is revealed for an STM imaging bias inside the oxide band gap and below the vacuum energy of the system. The contrast reverses at $\sim 3.5 \mathrm{~V}$, when the conductance through the ad-metal drops below the one through the bare oxide. The conductance switch reflects the opening of new electron-transport channels in or above the film, such as the oxide conduction band or low-lying vacuum states [25,29]. The contrast change is particularly strong at the perimeter of the nanoislands. While the brim appears brighter than the interior in the low-bias regime due to additional electronic states in the Au edge atoms [9], it turns dark when crossing the oxide conduction-band edge at higher bias. Moreover, the troughs around the $\mathrm{Au}$ islands are deeper and broader on $\mathrm{CaO}$ than $\mathrm{MgO}$ films. We will rationalize this effect later by a different degree of band bending in the two oxide films, as induced by the charged ad-gold.

\section{B. Electronic properties of the Au nanostructures}

Both $\mathrm{CaO}$ - and $\mathrm{MgO}$-supported $\mathrm{Au}$ islands exhibit a quantized electronic structure that governs point spectra as well as maps of the differential conductance $(d I / d V)$ [30]. Figure 3

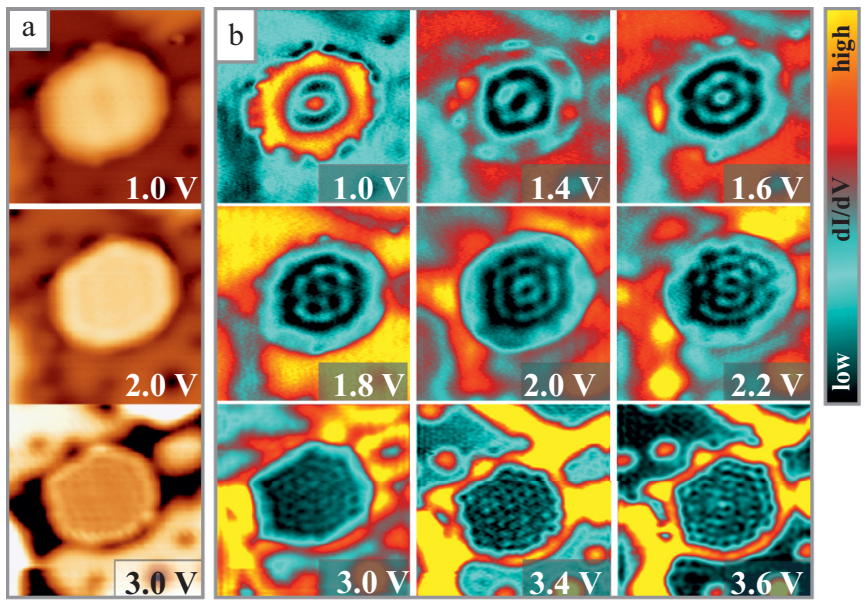

FIG. 3. (Color online) (a) Topographic and (b) conductance maps of an $\mathrm{Au}$ island on $\mathrm{MgO} / \mathrm{Ag}(001)$ taken at different bias voltages $\left(11 \times 11 \mathrm{~nm}^{2}\right)$. Note the bias-dependent evolution of a standing-wave pattern inside the island. shows a series of $d I / d V$ maps that probe the unoccupied state density at positive sample bias for an $\mathrm{Au} / \mathrm{MgO}$ island with $\sim 300$ atoms. Starting from 1.0 V, the initially featureless maps start to develop concentric rings that increase in number with bias voltage. Particularly nice examples are found at 1.0, 1.6, and $2.0 \mathrm{~V}$, when one, two, and three dark rings become visible around the center [Fig. 3(b)]. The maps also exhibit a radial fine structure, characterized by an increasing number of nodal planes cutting through the island center. Similar conductance patterns have been observed for metal ad-islands and hole structure on metallic [31-33] and oxide surfaces [34]. For Au nanostructures, they have been ascribed to standing-wave patterns arising from the confinement of an Au $6 s p$ electronic band. Level quantization in hexagonal potential wells with infinite walls can be described empirically with a linear relationship between eigenenergy $E_{n}$ and quantum number $n$ [31]: $E_{n}=E_{0}+\left(n / m_{\mathrm{eff}} \Omega^{*}\right) .{ }^{1}$ Here, $E_{0}$ and $m_{\text {eff }}$ denote the onset energy and the effective electron mass of the Au $6 s p$ band, respectively. To determine the energy position of the $\mathrm{Au}$ quantum well states (QWSs), we have fitted 1D cuts through the bias-dependent $d I / d V$ maps to idealized standing-wave patterns. The fitting procedure has been described in earlier work $[28,31,35]$, and we therefore focus on the results at this point.

For the MgO-supported Au island in Fig. 3, we find a linear dispersion of QWSs that give rise to the observed standingwave pattern. Its slope, accounting for the electron mobility in the manifold, amounts to $0.69 \mathrm{~V} \mathrm{~nm}$, while the onset position was determined to $0.1 \mathrm{~V}$ [Fig. 4(a)]. Both values are in good agreement with earlier results for monolayer $\mathrm{Au}$ islands on the $\mathrm{MgO}$ support [28]. The respective band can be located directly in electronic-structure calculations for bulk gold, performed by Häkkinen [36]. The $\mathrm{Au}(111)$ surface is hereby represented by a hexagonal plane around the $L$ point of the irreducible Brillouin zone. The band structure in Fig. 4(d) indeed contains a band of matching energy that proceeds from the $L$ point to the zone boundary. It is of $\mathrm{Au} 6 s p$ character, hence parabolic

${ }^{1}$ In Ref. [31], the dispersion is expressed as $E_{n}=E_{0}+\left(\lambda_{n} / m_{\mathrm{eff}} \Omega\right)$. The equation shown here was obtained by fitting the eigenvalues $\lambda_{n}$ with $\lambda_{n}=10.8 n+5.9$ and introducing a modified quantization area $\Omega^{*}$. 

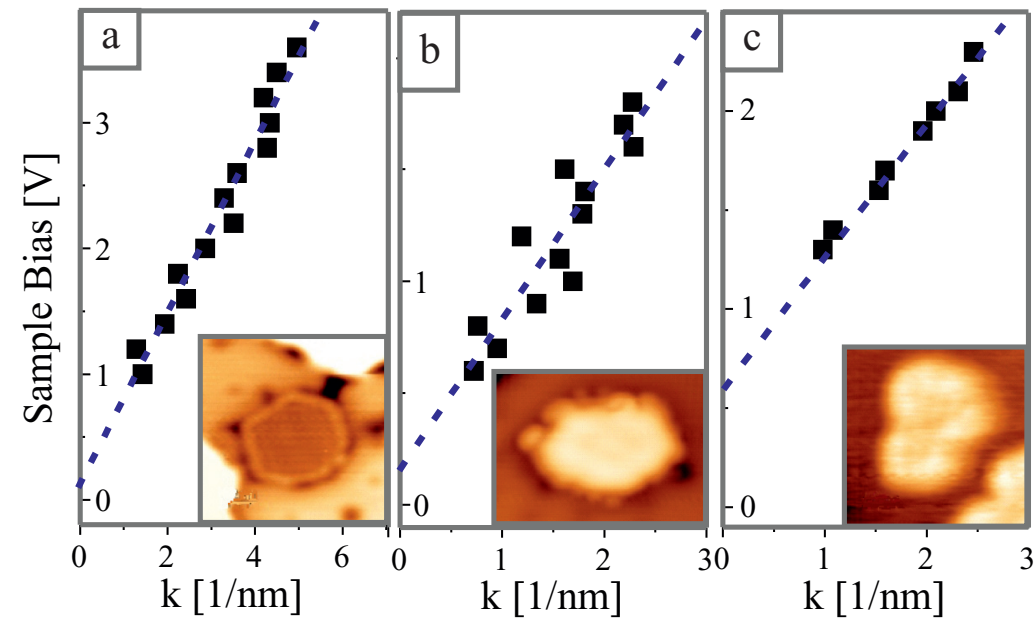

$\begin{array}{ll}\text { Slope/ } & 0.69 \mathrm{~V} \cdot \mathrm{nm} / 0.1 \mathrm{~V} \quad 0.66 \mathrm{~V} \cdot \mathrm{nm} / 0.2 \mathrm{~V} \quad 0.68 \mathrm{~V} \cdot \mathrm{nm} / 0.6 \mathrm{~V} \\ \text { Onset: }\end{array}$

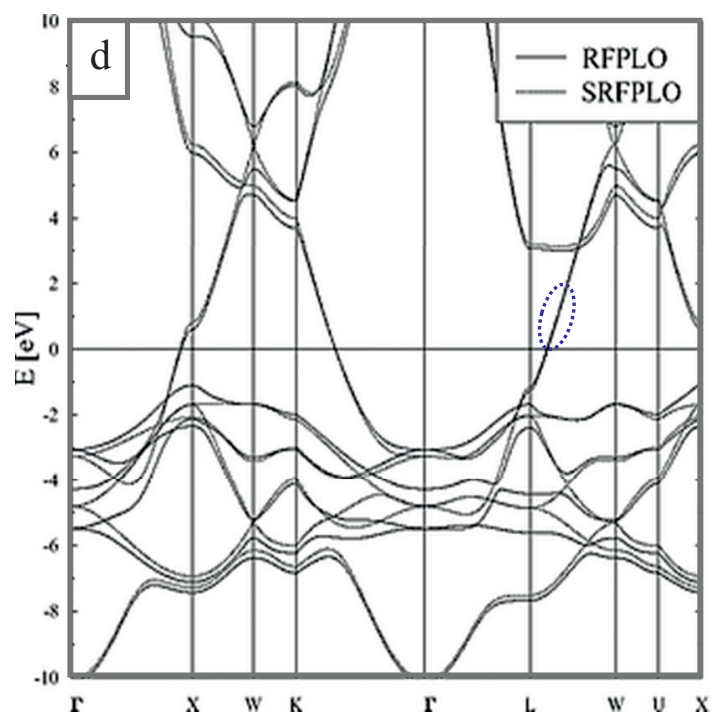

FIG. 4. (Color online) (a)-(c) Experimental dispersion relations of QWSs in three differently sized Au islands on MgO thin films. The number of atoms per island amounts to approximately (a) 300, (b) 200, and (c) 44. The respective islands are shown in the insets [(a) $3.4 \mathrm{~V}, 11 \times 11 \mathrm{~nm}^{2}$; (b) $0.5 \mathrm{~V}, 11 \times 8 \mathrm{~nm}^{2}$; (c) $-1.3 \mathrm{~V}, 6.5 \times 5.5 \mathrm{~nm}^{2}$ ]. Results for island (a) are based on the $d I / d V$ maps shown in Fig. 3. (d) Calculated band structure for bulk gold, taken from Ref. [36]. The electronic band that experiences in-plane quantization in the Au islands is marked by an ellipse. Despite the limited size of the nanostructures, the QWSs already reproduce main properties of the associated bulk band.

at the onset but increasingly flat near the $W$ point at the zone boundary. Its slope in the linear region $(\sim 0.6 \mathrm{~V} \mathrm{~nm})$ agrees well with the experimental value. Note that small deviations in the band dispersion are expected along different directions $[L W$ versus $L K]$ in the Brillouin zone that are not resolved in our data.

The main difference between experiment and theory concerns the band onset, which is well below the Fermi level in the calculation [36], but above $E_{F}$ in the experiment. We assign this discrepancy to a quantum size effect, as measured band onsets vary strongly with the island diameter. While the smallest island in Fig. 4 (44 atoms) has a band onset at $0.6 \mathrm{~V}$, it shifts to 0.2 and $0.1 \mathrm{~V}$ in islands with 200 and 300 atoms, respectively. Apparently, the depth of the quantum well still increases with increasing atom count and approaches the bulk value only gradually. Interestingly, the slope of the dispersion that reflects the overlap of adjacent electronic states is hardly affected by the system size. Also the monolayer nature of the island, hence the missing vertical dimension, seems unimportant for the in-plane electronic properties.

Gold islands on the doped $\mathrm{CaO}$ support have been analyzed in a similar fashion. Let us reiterate at this point that the $\mathrm{CaO}$ film is about 20 times thicker than the $\mathrm{MgO}$ layer and contains a considerable amount of Mo donors. Similar to $\mathrm{Au} / \mathrm{MgO}$, the ad-islands on $\mathrm{CaO}(001)$ display standing-wave patterns although their quality is lower due to the reduced electron transmissibility through the thick oxide film (Fig. 5). In fact, $d I / d V$ mapping could be performed only at bias voltages above the conduction band onset, while unstable tunneling conditions prevented any data acquisition inside the gap region. Also, nature and dispersion of the respective QWSs seem to deviate from those of the $\mathrm{Au} / \mathrm{MgO}$ system [Fig. 6(a)]. The band onset at $1.5 \mathrm{~V}$ is higher and the slope of $0.8 \mathrm{~V} \mathrm{~nm}$ is steeper than on the $\mathrm{MgO}$ support. Moreover, the dispersion is linear only up to a critical bias of $4.2 \mathrm{~V}$ and becomes constant beyond this value [Fig. 6(a)]. The kink in the $E(k)$ diagram occurs at $k=3.8 \mathrm{~nm}^{-1}$, which corresponds to the inverse periodicity of the moiré pattern. Apparently, the electron waves lock into the stripe pattern observed on the Au islands, or in other words, the moiré pattern creates a new zone boundary that restricts the $k$ vector of the respective electronic band $[37,38]$. Given the large differences in the electronic structure of $\mathrm{CaO}$ - and $\mathrm{MgO}$-supported $\mathrm{Au}$ islands, the question arises whether both systems indeed follow the same physical picture.

In the following paragraph, we demonstrate that the differences mainly arise from a better decoupling of $\mathrm{CaO}$-grown $\mathrm{Au}$ islands from the metal support, which renders them more sensitive to polarization in the tip-electric field. In general, STM experiments on insulating films involve electron transport through a double barrier junction, comprising a vacuum gap between tip and particle and an oxide gap between particle and conductive support [39]. The two barriers lead to a voltage
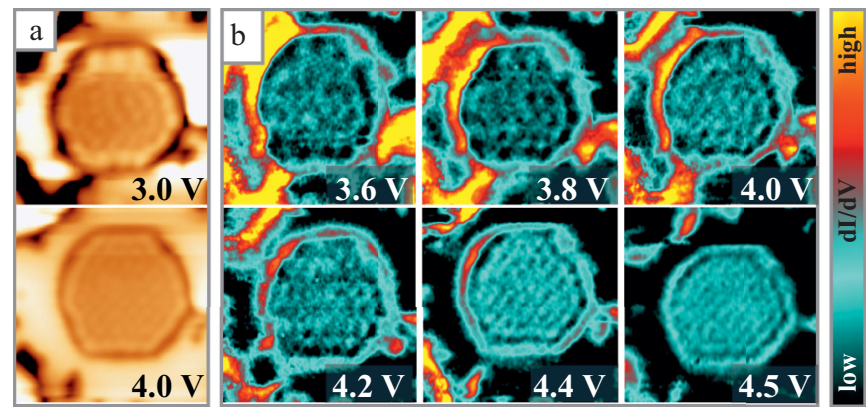

FIG. 5. (Color online) (a) Topographic and (b) conductance maps of an $\mathrm{Au}$ island on $25 \mathrm{ML} \mathrm{CaO}(001)$ taken at different bias voltages $\left(12 \times 12 \mathrm{~nm}^{2}\right)$. The evolution from standing waves to a bias-independent moiré pattern is clearly discernible. 

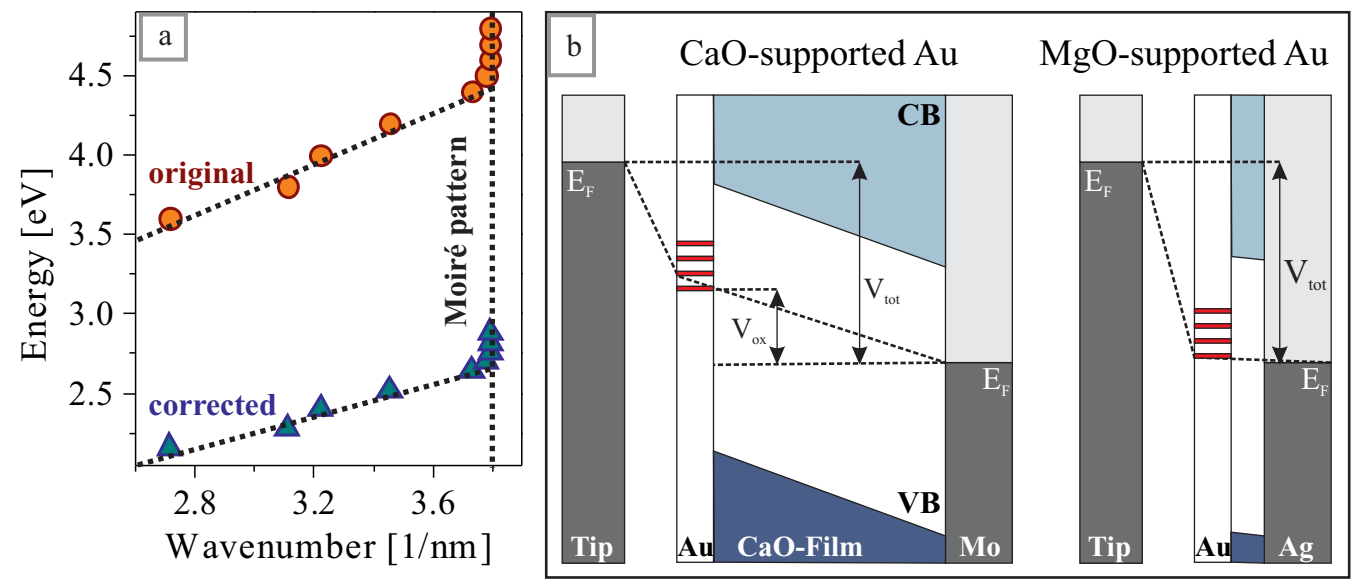

FIG. 6. (Color online) (a) Experimental dispersion relation of QWSs in the CaO-supported Au island shown in Fig. 5 (200 atoms). After correction for tip-induced band bending, the eigenstates shift down in energy and approach the values obtained for MgO-grown nanostructures. Above a critical $k$ of $3.8 \mathrm{~nm}^{-1}$, the wave pattern locks into the periodicity of the Moiré pattern and becomes constant. (b) Potential diagrams of STM junctions containing a thick $\mathrm{CaO}$ as well as a thin $\mathrm{MgO}$ film. Band-bending effects are relevant only for the polarizable $\mathrm{CaO}$ layer.

division $V_{\mathrm{vac}} / V_{\mathrm{ox}}$ in the STM junction that can be deduced from the thickness and dielectric constant of the gap material: $V_{\mathrm{ox}} / V_{\mathrm{vac}}=\left(d_{\mathrm{ox}} / \varepsilon_{\mathrm{ox}}\right)\left(\varepsilon_{\mathrm{vac}} / d_{\mathrm{vac}}\right)$ [40]. Due to this division, only a part of the applied bias actually drops inside the vacuum barrier, while a substantial fraction decays in the oxide spacer and induces a band bending towards the Fermi level of the tip [Fig. 6(b)]. The Au electronic system now floats between the two barriers and direct comparability between $\mathrm{MgO}$ - and $\mathrm{CaO}$-supported islands can be ensured only after correcting for this tip-induced band bending. To estimate the voltage drop inside the oxide, we have inserted the $\operatorname{MgO}(4 \AA)$ and $\mathrm{CaO}$ film thicknesses $(60 \AA)$ and the oxides dielectric constants $\left(\varepsilon_{\mathrm{ox}}=10\right)$ into the above equation. The width of the vacuum gap was approximated with $8 \AA$, neglecting bias-dependent variations in the tip-sample distance. The derived voltage drop in the ultrathin $\mathrm{MgO}$ film is negligible $\left(V_{\mathrm{ox}} / V_{\mathrm{vac}}=0.05\right)$, while roughly one-third of the total tip-sample bias actually drops inside the thick $\mathrm{CaO}$ spacer $\left(V_{\mathrm{ox}} / V_{\mathrm{vac}}=0.62\right)$.

After this renormalization, the electronic properties of $\mathrm{CaO}$-grown $\mathrm{Au}$ islands indeed converge against those of the $\mathrm{Au} / \mathrm{MgO}$ system [Fig. 6(a)]. While the band onset shifts downwards to $0.7 \mathrm{~V}$, the slope reduces to $0.55 \mathrm{~V} \mathrm{~nm}$. Note that error bars are still larger in the $\mathrm{CaO}$-based experiment, partly because of the fainter standing-wave patterns, partly because of uncertainties in the determination of the oxide thickness and the tip-sample distance. In this regard, we consider our experimental results on $\mathrm{MgO}$ - and $\mathrm{CaO}$-supported $\mathrm{Au}$ structures to be reasonably matched, suggesting that one and the same $\mathrm{Au}$ electronic band experiences confinement in both cases. So far, we have not clarified whether charge transfer into the Au ad-islands, as a central point of our study, occurs in a similar manner on both metal-oxide systems. We remind that the transfer electrons originate from bulk $\mathrm{Ag}(001)$ in the $\mathrm{MgO}$ case, but from Mo ions with donor character in the $\mathrm{CaO}$ films that are too thick for direct electron tunneling from the support. If the two charging routes indeed converge to the same final state, ultrathin oxide films could be used as model systems for bulk oxides that are difficult to explore due to their insulating nature $[1,2]$. In our final paragraph, we thus compile experimental evidence for the occurrence of electron transfer on $\mathrm{MgO}$ and $\mathrm{CaO}$ films, although the absolute charge state of the $\mathrm{Au}$ nanoislands could not be extracted from the experiment.

A first indication for charge-transfer processes comes from the observed growth shape of $\mathrm{Au}$ islands on the two oxide films. On both $\mathrm{MgO}$ and $\mathrm{CaO}$, gold aggregates into monolayer islands, while 3D particles are hardly found (Figs. 1 and 2). The $2 \mathrm{D}$ growth regime was safely connected with a charged nature of the ad-gold, and ascribed to an enhanced Coulomb and polaronic coupling between $\mathrm{Au}$ anions and the polarizable oxide support [3-5,7]. The 2D islands disappear if the thickness of the oxide spacer exceeds the electron tunneling length, or if no alternative electron sources, e.g., dopants, are available in the oxide matrix [20,25]. The predominance of monolayer $\mathrm{Au}$ islands thus verifies the robustness of charge transfer on both oxide supports.

A second hint for the charged nature of the ad-gold comes from the energy position of vacuum states in the vicinity of the metal islands. Vacuum states, also referred to as field-emission resonances (FERs) in the context of STM, show up as distinct conductance maxima in $d I / d V$ spectra taken at bias voltages above the sample work function [29]. The presence of excess surface charges now leads to characteristic shifts of the FERs; downward in electron-deficient structures and upward on electron-rich surfaces. The lowest resonance thereby marks the approximate position of the vacuum energy, while higher levels are additionally altered by the tip-electric field [41]. Figure 7(a) displays a series of FER spectra, measured on a path that runs from bare $\mathrm{CaO}$ to an $\mathrm{Au}$ island. Apparently, the FERs undergo a continuous upshift when moving towards the ad-gold, whereby the first resonance changes its position from 4.0 to $6.0 \mathrm{~V}$. This shift reflects the expected electron accumulation in the islands, but also relates to the intrinsically higher work function of gold with respect to the oxide film [25]. A similar trend is revealed for the $\mathrm{Au} / \mathrm{MgO}$ system [Fig. 7(b)]. In contrast to the $\mathrm{CaO}$ case, however, the transition between oxide and particle-governed FERs is more abrupt in accordance with the lower polarizability of the ultrathin $\mathrm{MgO}$ film. 

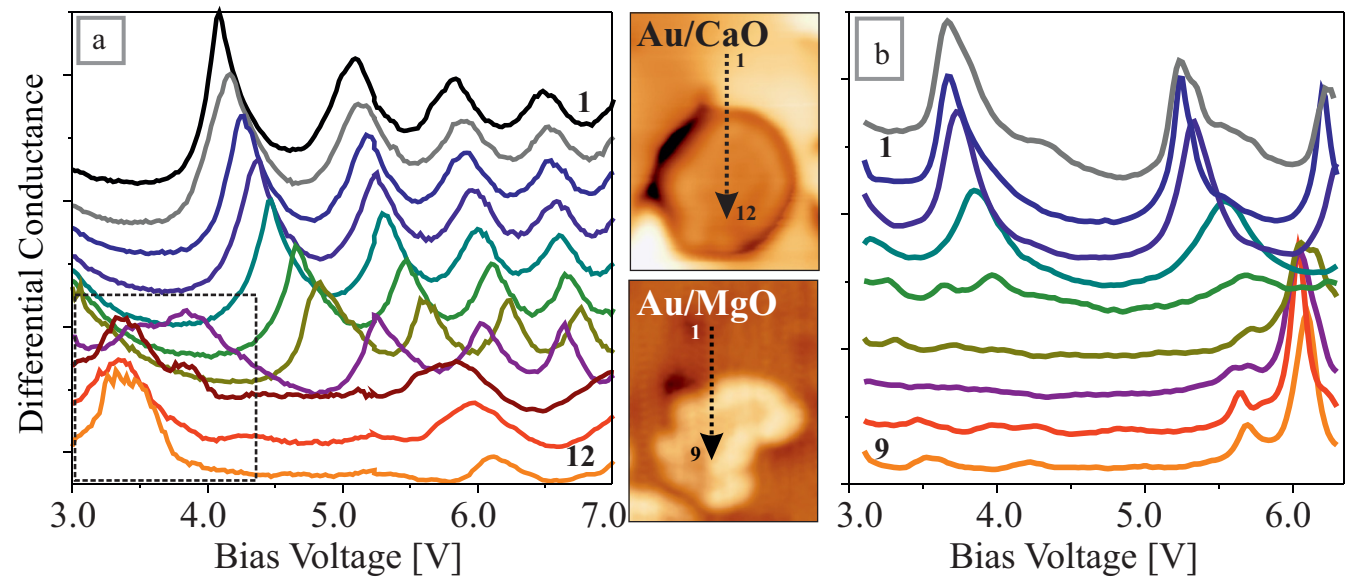

FIG. 7. (Color online) Series of $d I / d V$ spectra taken along a path from (a) bare $\mathrm{CaO}$ and (b) bare $\mathrm{MgO}$ to an $\mathrm{Au}$ island, as shown in the insets [(a) $3.5 \mathrm{~V}, 11 \times 25 \mathrm{~nm}^{2}$; (b) $1.7 \mathrm{~V}, 7 \times 15 \mathrm{~nm}^{2}$ ]. The spectra have been acquired with enabled feedback loop and $25 \mathrm{pA}$ set-point current. The high-bias $d I / d V$ oscillations are FERs that shift up in energy when approaching the negatively charged ad-gold. The spectral region marked with a dashed box in (a) displays the Au QWSs, uncorrected for the effect of tip-induced band bending.

A final indication for charge accumulation in the nanogold is derived from the negative STM image contrast around the ad-islands at high bias [Figs. 3(a) and 8(a) for $\mathrm{MgO}$ and $\mathrm{CaO}$, respectively]. As discussed before, the extra electrons localize mainly along the island perimeter, a distribution that maximizes their mutual distance and minimizes the impact of electron-electron repulsion inside the nanostructure [9]. Moreover, the low-coordinated edge atoms contain empty states that can be easily populated with the transfer electrons from the support. The dark appearance of the island perimeter
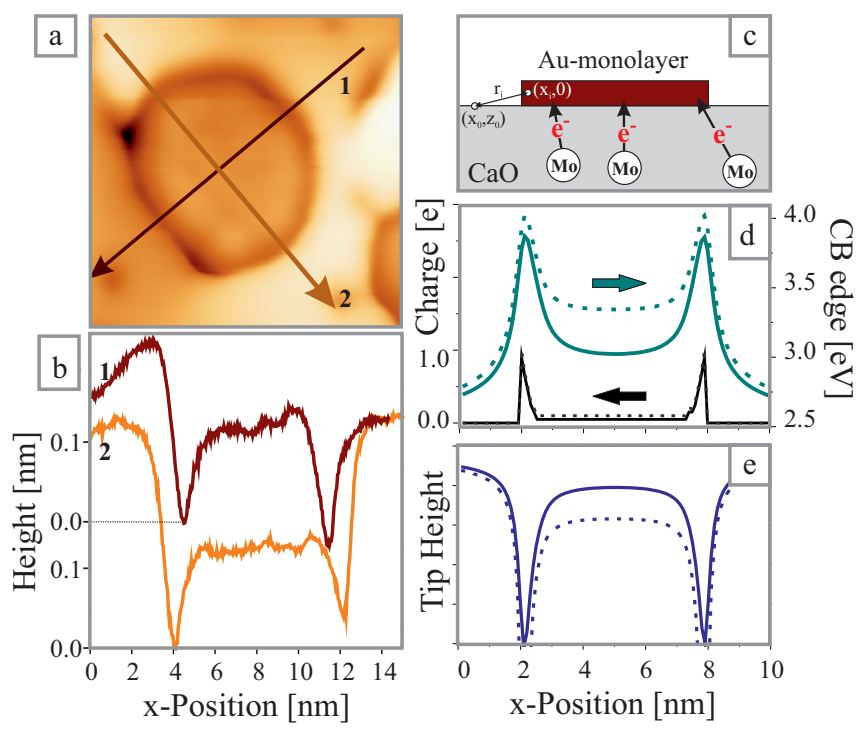

FIG. 8. (Color online) (a) STM image $\left(3.5 \mathrm{~V}, 12 \times 12 \mathrm{~nm}^{2}\right)$ and (b) corresponding height profiles across an Au monolayer island on $\mathrm{CaO}(001)$. (c) $1 \mathrm{D}$ model and (d) assumed charge distribution within the island. The exclusive appearance of edge electrons and a mixture of edge and inner charges $(0.1 e)$ are depicted by solid and dashed lines, respectively. The resulting shift of the oxide conduction band is shown in turquoise for both scenarios. (e) Simulated height of the STM tip above a charged Au island; the pronounced negative contrast at the metal-oxide boundary is clearly discernible. now reflects a polarization of the oxide layer by these extra electrons, as illustrated with the following model. The uneven electron distribution in the $\mathrm{Au}$ island $q_{i}\left(x_{i}\right)$ is simulated with a simple 1D profile, in which edge atoms are negatively charged while inner atoms are neutral [Fig. 8(d)]. Given the poor screening, electronic states in the insulating oxide respond to these extra electrons by an upward bending, which can be modeled with a screened Coulomb interaction: $V\left(x_{0}\right) \propto \sum_{i}\left(q_{i} / \varepsilon_{0} \varepsilon_{\mathrm{CaO}} r_{i}\right)$ [11]. Here, $r_{i}=\sqrt{\left(x_{0}-x_{i}\right)^{2}+z_{0}^{2}}$ describes the distance between an excess electron in the gold and a position in the oxide film $\left(z_{0} \sim 2.5 \AA\right.$ for the vertical separation between $\mathrm{Au}$ ad-layer and first oxide plane). The obtained upshift of the conduction band peaks directly below the electron-rich perimeter and decays gradually towards the interior of the Au island and the oxide film.

The STM image contrast can now be modeled from this spatial variation of the band edges. At high positive bias as used for data acquisition, the conduction band provides most of the final states for electrons from the STM tip. The stabilizing current $I$ is thus proportional to that part of the conduction band that lies below the set-point voltage $V_{s}: I(V) \propto$ $e^{-\kappa z} \rho_{\text {Tip }} \int_{\mathrm{VCB}}^{\mathrm{VS}} \rho_{\mathrm{CaO}} d E \propto \rho_{\mathrm{Tip}} \rho_{O} e\left(V_{S}-V_{C B}\right) e^{-\kappa z}$. Here, $\rho_{\text {tip }}$ and $\rho_{\mathrm{CaO}}$ are the state densities in tip and oxide film, while $\kappa$ is the decay constant of wave functions into the vacuum that can be determined directly from experimental current-distance plots [42]. Solving the formula for $z$ gives a qualitative expression for the tip-sample distance when scanning across a negatively charged island: $z \propto(1 / \kappa) \ln \left[I(V) / \rho_{\text {Tip }} \rho_{O} e\left(V_{S}-V_{C B}\right)\right]$ [Fig. 8(e)]. Absolute values cannot be given at this point due to uncertainties in the state density and the exponential decay constant $\kappa$.

Our simulation reproduces the decreasing tip height in the vicinity of $\mathrm{Au}$ islands, now assigned to an upshift of the oxide bands in response to the extra electrons in the gold [34]. The STM contrast is lowest directly at the boundary, where the state density accessible for tip electrons has a minimum, and takes an intermediate value inside the island. Our height simulation qualitatively captures the experimental behavior and can thus be considered as additional evidence for a charge 
accumulation at the island perimeter. The extension of the negative-contrast region from the island edge towards the oxide surface depends on the polarizability of the oxide film, hence on its thickness and dielectric constant. Experimentally, the dark regions around the ad-islands are more pronounced on $\mathrm{CaO}$, where they reach up to $3 \mathrm{~nm}$ into the bare oxide surface (compared to $1 \mathrm{~nm}$ for the $\mathrm{Au} / \mathrm{MgO}$ system). As the static dielectric constant is similar for the two oxides, the effect is mainly governed by the ultrathin nature of the $\mathrm{MgO}$ and the decisive influence of the metal support. Consequently, the spatial extension of the negative-contrast region cannot be exploited to determine the number of excess electrons in the ad-islands from the experiment. Our model confirms the general occurrence of charge transfer on $\mathrm{CaO}$ and $\mathrm{MgO}$ films, but is unable to provide quantitative insight into its efficiency.

\section{CONCLUSIONS}

Both thin $\mathrm{MgO}$ and thick $\mathrm{CaO}$ films doped with $\mathrm{Mo}$ donors were found to induce electron-transfer processes into ad-gold bound to their surfaces. Experimental indications for the charge exchange are the monolayer nature of the $\mathrm{Au}$ particles due to a reinforced metal-oxide adhesion, the negative image contrast, and the upshift of vacuum states around the ad-gold, as triggered by the extra electrons at the island perimeter. The electronic structure of the $\mathrm{Au}$ islands is governed by quantized electronic states that give rise to bias-dependent standing-wave patterns. Within the accuracy of our measurements, we find identical properties of $\mathrm{MgO}$ and $\mathrm{CaO}$-grown particles, suggesting a similar nature of the binding and charge-transfer characteristics on both oxide supports. Evidently, the two charging routes explored here, i.e., electron transfer from a bulk metal through an ultrathin oxide spacer and charge donation from donor-type impurities dispersed in an oxide matrix, give rise to a comparable response of the $\mathrm{Au}$ deposits. Given this correspondence, we argue that within certain limits ultrathin oxide films can be used to model insulating bulk oxides in surface science experiments. Our conclusion emphasizes the significance of atomic scale experiments to bridge model studies in heterogeneous catalysts with technical applications.

\section{ACKNOWLEDGMENTS}

We gratefully acknowledge financial support from the DFG Cluster of Excellence "Unicat" and the COST Action "Reducible Oxides." Y.C. and C.S. acknowledge support from the Humboldt Foundation and the Studienstiftung des Deutschen Volkes, respectively. The authors thank W.-D. Schneider for insightful discussions.

Y.C. and C.S. contributed equally to this work.
[1] N. Nilius, T. Risse, S. Shaikhutdinov, M. Sterrer, and H.-J. Freund, in Gold Clusters, Colloids and Nanoparticles II, edited by D. M. P. Mingos (Springer International Publishing, Cham, 2014), p. 91.

[2] L. Giordano and G. Pacchioni, Accounts. Chem. Res. 44, 1244 (2011).

[3] M. Sterrer, T. Risse, M. Heyde, H.-P. Rust, and H.-J. Freund, Phys. Rev. Lett. 98, 206103 (2007).

[4] D. Ricci, A. Bongiorno, G. Pacchioni, and U. Landman, Phys. Rev. Lett. 97, 036106 (2006).

[5] N. Mammen, S. Narasimhan, and S. D. Gironcoli, J. Am. Chem. Soc. 133, 2801 (2011).

[6] C. R. Henry, Surf. Sci. Rep. 31, 235 (1998).

[7] G. Pacchioni, L. Giordano, and M. Baistrocchi, Phys. Rev. Lett. 94, 226104 (2005).

[8] P. Frondelius, H. Häkkinen, and K. Honkala, New J. Phys. 9, 339 (2007).

[9] X. Lin, N. Nilius, M. Sterrer, P. Koskinen, H. Häkkinen, and H.-J. Freund, Phys. Rev. B 81, 153406 (2010).

[10] V. Simic-Milosevic, M. Heyde, X. Lin, T. König, H.-P. Rust, M. Sterrer, T. Risse, N. Nilius, H.-J. Freund, L. Giordano, and G. Pacchioni, Phys. Rev. B 78, 235429 (2008).

[11] N. Nilius, M.V. Ganduglia-Pirovano, V. Brázdová, M. Kulawik, J. Sauer, and H.-J. Freund, Phys. Rev. B 81, 045422 (2010).

[12] U. Kreibig, M. Gartz, and A. Hilger, Ber. Bunsen-Ges. 101, 1593 (1997).

[13] P. Frondelius, H. Häkkinen, and K. Honkala, Angew. Chem., Int. Ed. 49, 7913 (2010).

[14] A. A. Herzing, C. J. Kiely, A. F. Carley, P. Landon, and G. J. Hutchings, Science 321, 1331 (2008).
[15] S. Mostafa, F. Behafarid, J. R. Croy, L. K. Ono, L. Li, J. C. Yang, A. I. Frenkel, and B. R. Cuenya, J. Am. Chem. Soc. 132, 15714 (2010).

[16] M. S. Chen and D. W. Goodman, Science 306, 252 (2004).

[17] F. Calaza, C. Stiehler, Y. Fujimori, M. Sterrer, S. Beeg, M. Ruiz-Oses, N. Nilius, M. Heyde, T. Parvianinen, K. Honkala, H. Häkkinen, and H.-J. Freund, Angew. Chem., Int. Ed. (2015), doi:10.1002/anie.201501420.

[18] L. Giordano, G. Pacchioni, J. Goniakowski, N. Nilius, E. D. L. Rienks, and H.-J. Freund, Phys. Rev. Lett. 101, 026102 (2008).

[19] L. Giordano, F. Cinquini, and G. Pacchioni, Phys. Rev. B 73, 045414 (2006).

[20] X. Shao, S. Prada, L. Giordano, G. Pacchioni, N. Nilius, and H. J. Freund, Angew. Chem., Int. Ed. 50, 11525 (2011).

[21] Z. Hu, B. Li, X. Y. Sun, and H. Metiu, J. Phys. Chem. C 115, 3065 (2011).

[22] X. Shao, N. Nilius, and H. J. Freund, J. Am. Chem. Soc. 134, 2532 (2012).

[23] X. Shao, P. Myrach, N. Nilius, and H. J. Freund, J. Phys. Chem. C 115, 8784 (2011); X. Shao, P. Myrach, N. Nilius, H. J. Freund, U. Martinez, S. Prada, L. Giordano, and G. Pacchioni, Phys. Rev. B 83, 245407 (2011).

[24] S. Schintke, S. Messerli, M. Pivetta, F. Patthey, L. Libioulle, M. Stengel, A. De Vita, and W.-D. Schneider, Phys. Rev. Lett. 87, 276801 (2001)

[25] X. Shao, N. Nilius, and H. J. Freund, Phys. Rev. B 85, 115444 (2012).

[26] J. Andersin, J. Nevalaita, K. Honkala, and H. Häkkinen, Angew. Chem., Int. Ed. 52, 1424 (2013). 
[27] J. Wollschläger, J. Viernow, C. Tegenkamp, D. Erdös, K. M. Schröder, and H. Pfnür, Appl. Surf. Sci. 142, 129 (1999).

[28] C. Stiehler, Y. Pan, W.-D. Schneider, P. Koskinen, H. Häkkinen, N. Nilius, and H.-J. Freund, Phys. Rev. B 88, 115415 (2013).

[29] G. Binnig, K. H. Frank, H. Fuchs, N. Garcia, B. Reihl, H. Rohrer, F. Salvan, and A. R. Williams, Phys. Rev. Lett. 55, 991 (1985).

[30] A. Mans, J. H. Dil, A. R. H. F. Ettema, and H. H. Weitering, Phys. Rev. B 66, 195410 (2002).

[31] J. Li, W.-D. Schneider, R. Berndt, and S. Crampin, Phys. Rev. Lett. 80, 3332 (1998).

[32] J. Lagoute, X. Liu, and S. Fölsch, Phys. Rev. Lett. 95, 136801 (2005).

[33] K. Schouteden and C. Van Haesendonck, Phys. Rev. Lett. 108, 076806 (2012)

[34] X. Lin, N. Nilius, H. J. Freund, M. Walter, P. Frondelius, K. Honkala, and H. Häkkinen, Phys. Rev. Lett. 102, 206801 (2009).
[35] N. Nilius, T. M. Wallis, and W. Ho, Science 297, 1853 (2002).

[36] H. Häkkinen, Chem. Soc. Rev. 37, 1847 (2008).

[37] J. Repp, G. Meyer, and K.-H. Rieder, Phys. Rev. Lett. 92, 036803 (2004).

[38] Y. Pan, S. Benedetti, N. Nilius, and H.-J. Freund, Phys. Rev. B 84, 075456 (2011).

[39] S. W. Wu, G. V. Nazin, X. Chen, X. H. Qui, and W. Ho, Phys. Rev. Lett. 93, 236802 (2004).

[40] R. M. Feenstra, Phys. Rev. B 50, 4561 (1994); G. J. de Raad, D. M. Bruls, P. M. Koenraad, J. H. Wolter, ibid. 66, 195306 (2002).

[41] O. Y. Kolesnychenko, Y. A. Kolesnichenko, O. I. Shklyarevskii, and H. van Kempen, Physica B (Amsterdam, Neth.) 291, 246 (2000).

[42] J. C. Chen, Introduction to Scanning Tunneling Microscopy (Oxford University Press, New York, 1993). 